Please do not remove this page

RMIT

UNIVERSITY

\title{
Optimising a nonlinear utility function in multi-objective integer programming
}

Ozlen, Melih; Azizoglu, Meral; Burton, Benjamin

https://researchrepository.rmit.edu.au/esploro/outputs/9921859391301341/filesAndLinks?institution=61RMIT_INST\&\&index=null

Ozlen, M., Azizoglu, M., \& Burton, B. (2012). Optimising a nonlinear utility function in multi-objective integer programming. Journal of Global Optimization, 56, 93-102.

https://doi.org/10.1007/s10898-012-9921-4

Document Version: Accepted Manuscript

Published Version: https://doi.org/10.1007/s10898-012-9921-4

Repository homepage: https://researchrepository.rmit.edu.au

(c) Springer Science+Business Media, LLC. 2012

Downloaded On 2023/04/26 13:13:19 +1000

Please do not remove this page 
Thank you for downloading this document from the RMIT Research Repository.

The RMIT Research Repository is an open access database showcasing the research outputs of RMIT University researchers.

RMIT Research Repository: http://researchbank.rmit.edu.au/

\section{Citation:}

Ozlen, M, Azizoglu, M and Burton, B 2013, 'Optimising a nonlinear utility function in multi-objective integer programming', Journal of Global Optimization, vol. 56, no. 1, pp. 93-102.

See this record in the RMIT Research Repository at:

https://researchbank.rmit.edu.au/view/rmit:20718

Version: Accepted Manuscript

Copyright Statement:

C) Springer Science+Business Media, LLC. 2012

\section{Link to Published Version:}

http://dx.doi.org/10.1007/s10898-012-9921-4 


\title{
Optimising a nonlinear utility function in multi-objective integer programming
}

\author{
Melih Ozlen, Meral Azizoğlu, Benjamin A. Burton
}

\begin{abstract}
In this paper we develop an algorithm to optimise a nonlinear utility function of multiple objectives over the integer efficient set. Our approach is based on identifying and updating bounds on the individual objectives as well as the optimal utility value. This is done using already known solutions, linear programming relaxations, utility function inversion, and integer programming. We develop a general optimisation algorithm for use with $k$ objectives, and we illustrate our approach using a tri-objective integer programming problem.
\end{abstract}

Keywords: Multiple objective optimisation, integer programming, nonlinear utility function

\section{Introduction}

The majority of studies reported in the optimisation literature consider a single objective, such as minimising cost or maximising profit. However, in practice, there are usually many objectives that need to be considered simultaneously. In particular, the increasing effect of globalisation brings safety, environmental impact and sustainability issues, and hence their related performance measures, into consideration. The practical aim is to find solutions that are not only profitable but also safe, green and sustainable.

Multi-Objective Integer Programming (MOIP) considers discrete feasible sets defined by integer variables. The main focus of literature on MOIP has been on enumerating the entire integer nondominated set, or on optimising a single linear utility function. However, many practical situations require the optimisation of a nonlinear utility function that combines multiple objectives into one. Prominent applications of such problems include, but are not limited to, pricing, routing, production planning, resource allocation, portfolio selection, capital budgeting, and designing networks. In such applications a utility function can capture precisely the decision maker's preferences for how to balance conflicting objectives, such as cost versus environmental impact in routing problems, or profit versus risk in portfolio selection.

Besides their practical importance, these optimisation problems are theoretically challenging as they - even their simpler single-objective versions - fall into the class of NP-hard problems. Despite their practical and theoretical importance, there is no reported research on such problems, at least to the best of our knowledge. Recognising this gap in the literature, in this paper we address the optimisation of an explicitly defined nonlinear utility function of multiple objectives over the integer efficient set, and provide a general framework for its resolution. 
Throughout this paper we assume that the utility function is strictly increasing with respect to each individual objective. Such monotonicity is standard for a utility function, whose role is to combine many individual objectives into a single global "utility objective".

A MOIP problem defines a discrete feasible set of efficient points and corresponding nondominated objective vectors, and the optimal value of our nonlinear utility function can always be found in this nondominated set. A naïve solution to our optimisation problem could therefore be to generate all nondominated objective vectors for the MOIP problem, and then to evaluate the utility function in each case. However, this naïve approach would be highly impractical, since the number of nondominated vectors can be extremely large in general.

Recognising this, we develop a more sophisticated approach to optimise a nonlinear utility function over the integer efficient set, in which we generate only a smaller subset of nondominated objective vectors. To avoid the generation of unpromising solutions, we compute objective optimality bounds by combining the best solution found so far with the nonlinear utility function. To generate the subset of promising nondominated objective vectors, we use an algorithm by Özlen and Azizoğlu [2009] that recursively solves MOIP problems with fewer objectives.

The rest of the paper is organised as follows. Section 2 reviews the related literature. In Section 3 we explain our algorithm in full and prove its correctness. Section 4 offers a detailed illustration of the workings of the algorithm, using an instance of a tri-objective integer programming problem. We conclude in Section 5

\section{Literature}

The best-studied cases of MOIP are Multi-Objective Combinatorial Optimisation (MOCO) problems. These are special cases of MOIP that have special constraint set structures. Ehrgott and Gandibleux 2000, 2004 provide rich surveys of MOCO studies that use exact and approximate approaches, respectively. They address some special problem structures and discuss their solution methodologies. Ehrgott and Gandibleux [2002] survey other MOCO problems including, but not limited to, nonlinear programming, scheduling and multi-level programming. These recent studies show a considerable growth in the MOCO literature. However, research on generating all nondominated objective vectors for MOIP, or on optimisation over that set, is still scarce.

Klein and Hannan [1982] develop an approach based on the sequential solutions of the single-objective Integer Programming (IP) problems. Their algorithm generates a subset, but not necessarily the whole set, of all nondominated objective vectors. Sylva and Crema [2004] improve the approach of Klein and Hannan [1982] by defining a weighted combination of all objectives, and their approach guarantees to generate all nondominated objective vectors.

Klamroth et al. 2004] and Ehrgott 2006] study the general MOIP problem. Klamroth et al. 2004] discuss the importance of using upper bounds on the objective function values when generating the nondominated set, and define composite functions to obtain such bounds. To form the composite functions, they propose classical optimisation methods such as cutting plane and branch and bound. Ehrgott [2006] discusses various scalarisation techniques, and proposes a generalised technique that encompasses the others as special cases. He also proposes an elastic constraint method to identify all nondominated points, whose power is dependent on the existence of sophisticated problem-dependent methods for solving the single objective version. 
Özlen and Azizoğlu [2009] develop a general approach to generate all nondominated objective vectors for the MOIP problem, by recursively identifying upper bounds on individual objectives using problems with fewer objectives. Dhaenens et al. [2010] present a similar but parallel algorithm for MOCO problems, that again involves recursively solving problems with fewer objectives. Przybylski et al. [2010b] and Özpeynirci and Köksalan [2010] propose similar algorithms to identify all extreme nondominated objective vectors for a MOIP problem. They both utilise a weighted single-objective function and partition the weight space to identify the set of extreme supported nondominated objective vectors, adapting a method first proposed for Multi-Objective Linear Programming (MOLP) by Benson and Sun [2000, 2002]. Przybylski et al. [2010a] extend the two phase method, originally developed to generate the nondominated set for bi-objective problems, to handle problems with three or more objectives.

There are some recent studies proposing preference-based methods for specific MOCO problems. Le Huédé et al. [2006] optimise a utility function in Multi-Attribute Utility Theory (MAUT) that is composed of piecewise-linear utility functions and a Choquet integral in a constraint programming-based algorithm. Perny and Spanjaard [2005] propose algorithms that consider any preorder for a multiobjective minimum weight spanning tree problem. For the same problem, Galand et al. [2010a] propose a branch and bound algorithm minimising a utility function composed of a concave Choquet integral and a partial convex utility function. Galand et al. [2010b] also propose a branch and bound algorithm maximising a convex Choquet integral function for the multiobjective knapsack problem. Contrary to these preferencebased studies, the method we propose in this paper is not problem-specific: it can be used with any MOIP problem, and requires no particular assumption about the utility function beyond the natural assumption of monotonicity.

There are few studies dealing with general MOIP problems in which the aim is to optimise a function. Abbas and Chaabane [2006] and Jorge [2009] deal with optimising a linear function over the efficient set of a MOIP problem.

\section{The algorithm}

In its general form, our algorithm optimises a nonlinear utility function of $k$ objectives over the integer programming efficient set. This problem can be defined precisely as:

Min $G\left(f_{1}(x), f_{2}(x), \ldots, f_{k}(x)\right)$

s.t. $x \in X$,

where $X$ is the set of feasible points defined by $A x=b, x_{j} \geq 0$ and $x_{j} \in \mathbb{Z}$ for all $j \in$ $\{1,2, \ldots, n\}$.

The individual objectives are defined as $f_{1}(x)=\sum_{j=1}^{n} c_{1 j} x_{j}, f_{2}(x)=\sum_{j=1}^{n} c_{2 j} x_{j}, \ldots$, and $f_{k}(x)=\sum_{j=1}^{n} c_{k j} x_{j}$, where $c_{i j} \in \mathbb{Z}$ for all $i \in\{1,2, \ldots, k\}$ and $j \in\{1,2, \ldots, n\}$. The nonlinear utility function $G\left(f_{1}(x), f_{2}(x), \ldots, f_{k}(x)\right)$ is assumed to be continuous and strictly increasing in each individual objective function $f_{1}(x), f_{2}(x), \ldots, f_{k}(x)$.

We refer to $G$ as a utility function because it combines the multiple objectives $f_{1}, \ldots, f_{k}$. However, we minimise $G$ for consistency with other authors such as Klein and Hannan [1982] and Przybylski et al. [2010a].

A point $x^{\prime} \in X$ is called $k$-objective efficient if and only if there is no $x \in X$ such that $f_{i}(x) \leq f_{i}\left(x^{\prime}\right)$ for each $i \in\{1,2, \ldots, k\}$ and $f_{i}(x)<f_{i}\left(x^{\prime}\right)$ for at least one $i$. The resulting objective vector $\left(f_{1}\left(x^{\prime}\right), f_{2}\left(x^{\prime}\right), \ldots, f_{k}\left(x^{\prime}\right)\right)$ is said to be $k$-objective nondominated. 
One typically works in objective space instead of variable space, since each nondominated objective vector might correspond to a large number of efficient points in variable space.

A point $x^{\prime} \in X$ is called optimal if and only if there is no $x \in X$ for which $G\left(f_{1}(x), f_{2}(x)\right.$, $\left.\ldots, f_{k}(x)\right)<G\left(f_{1}\left(x^{\prime}\right), f_{2}\left(x^{\prime}\right), \ldots, f_{k}\left(x^{\prime}\right)\right)$. Because our utility function is strictly increasing, any optimal point must also be $k$-objective efficient in variable space, and must yield a $k$ objective nondominated vector in objective space.

Our proposed method of finding an optimal point is based on a shrinking set of bounds for the $k$ individual objectives. We update the lower bounds using linear programming relaxations. Where possible, we update the upper bounds by using the lower bounds and inverting the utility function; where necessary, we update the upper bounds using the algorithm of Özlen and Azizoğlu [2009]. The shrinking bounds allow us to avoid unpromising portions of the integer efficient set, and the method of updating these bounds is designed to solve integer programs only when absolutely necessary.

Algorithm 1 gives the stepwise description of our procedure to find an optimal point for the nonlinear utility function $G\left(f_{1}(x), f_{2}(x), \ldots, f_{k}(x)\right)$. Key variables that we use in this algorithm include:

- $G^{B E S T}$, the best known value of the utility function;

- $f_{i}^{L B}$ and $f_{i}^{U B}$, the current lower and upper bounds on the values of the individual objective functions.

Algorithm 1 terminates with a $G^{B E S T}$ value that is the minimum of $G\left(f_{1}(x), f_{2}(x), \ldots, f_{k}(x)\right)$ among all efficient points; moreover, each time we update $G^{B E S T}$ we can record the corresponding efficient $x \in X$. Stated formally:

Theorem 1. Algorithm 1 finds the minimum value of $G\left(f_{1}(x), f_{2}(x), \ldots, f_{k}(x)\right)$ among all integer efficient points for the MOIP problem, and also identifies a corresponding $x \in X$ that attains this minimum.

Proof. As the algorithm runs we maintain the following invariants:

- The utility $G^{B E S T}$ is obtainable; that is, $G^{B E S T}=G\left(f_{1}(x), f_{2}(x), \ldots, f_{k}(x)\right)$ for some feasible integer point $x \in X$.

- Either $G^{B E S T}$ is already equal to the optimal utility, or else the optimal utility can be achieved for some point $x \in X$ with $f_{i}^{L B} \leq f_{i}(x) \leq f_{i}^{U B}$ for each objective $i \in\{1, \ldots, k\}$.

In essence, the lower and upper bounds $f_{i}^{L B}$ and $f_{i}^{U B}$ are used to bound the region of the integer efficient set that remains to be examined. It is easy to see that these invariants hold:

- In Step 0, each $f_{i}^{U B}$ is $\infty$, each $f_{i}^{L B}$ is the global minimum for $f_{i}(x)$, and $G^{B E S T}$ is obtained from a known point $x \in X$.

- In Step 1, any point $x \in X$ with $f_{i}(x)>f_{i}^{A}$ must have a utility worse than $G^{B E S T}$. This is because $G$ is strictly increasing, and so any such $x$ must satisfy $G\left(f_{1}(x), \ldots, f_{i}(x), \ldots\right.$, $\left.f_{k}(x)\right)>G\left(f_{1}^{L B}(x), \ldots, f_{i}^{A}, \ldots, f_{k}^{L B}(x)\right)$. The revised upper bound of $\left\lfloor f_{i}^{A}\right\rfloor$ is valid because each $f_{i}(x) \in \mathbb{Z}$. 


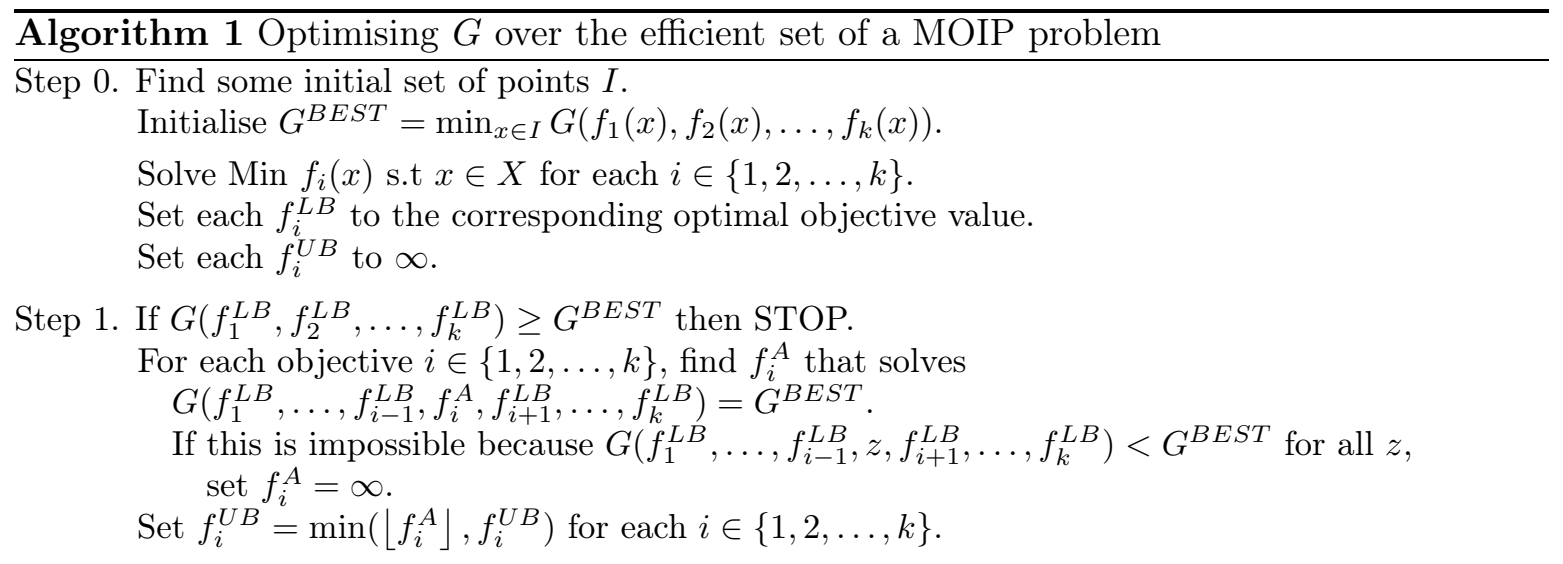

Step 2. For each objective $i \in\{1,2, \ldots, k\}$ :

Solve the LP relaxation of $\operatorname{Min} f_{i}(x)$ s.t. $x \in X, f_{1}(x) \leq f_{1}^{U B}, f_{2}(x) \leq f_{2}^{U B}, \ldots$, and $f_{k}(x) \leq f_{k}^{U B}$.

Let $f_{i}^{L P}=f_{i}\left(x^{*}\right)$ be the optimal objective value and $x^{*}$ be the corresponding optimal solution, and set $f_{i}^{L B}=\left\lceil f_{i}^{L P}\right\rceil$.

If $x^{*}$ is integer and $G\left(f_{1}\left(x^{*}\right), f_{2}\left(x^{*}\right), \ldots, f_{k}\left(x^{*}\right)\right)<G^{B E S T}$, set $G^{B E S T}=G\left(f_{1}\left(x^{*}\right), f_{2}\left(x^{*}\right), \ldots, f_{k}\left(x^{*}\right)\right)$.

If the lower bound $f_{i}^{L B}$ is updated for any objective $i$ then go to Step 1 .

Step 3. Use Özlen and Azizoğlu [2009] to update the upper bound $f_{k}^{U B}$. Specifically:

Begin generating all nondominated objective vectors for the $(k-1)$-objective MOIP problem Min $f_{1}(x), f_{2}(x), \ldots, f_{k-1}(x)$ s.t. $x \in X, f_{1}(x) \leq f_{1}^{U B}, f_{2}(x) \leq f_{2}^{U B}, \ldots, f_{k}(x) \leq f_{k}^{U B}$.

Each time we generate a $(k-1)$-objective vector, fix the first $k-1$ objectives to the values found and minimise $f_{k}(x)$. This two-step "lexicographic optimisation" yields a $k$-objective nondominated objective vector for the original problem.

If no feasible point exists then STOP.

Each time we generate a nondominated $k$-objective vector $f^{*}$, test whether $G\left(f_{1}^{*}, f_{2}^{*}, \ldots, f_{k}^{*}\right)<G^{B E S T}$. If true, set $G^{B E S T}=G\left(f_{1}^{*}, f_{2}^{*}, \ldots, f_{k}^{*}\right)$ and go to Step 1 immediately.

Let $S$ be the set of all nondominated objective vectors that were generated above. Set $f_{k}^{U B}=\max _{f \in S} f_{k}-1$ and go to Step 2 . 
- In Step 2, the revised lower bounds are valid because each optimal LP value $f_{i}^{L P}$ is equal to or better than the corresponding optimal IP value. Again we can round $\left\lceil f_{i}^{L P}\right\rceil$ because each $f_{i}(x) \in \mathbb{Z}$.

- In Step 3, any revision to $G^{B E S T}$ is valid because it comes from an efficient point: in particular, Özlen and Azizoğlu [2009] show that each solution $x \in X$ to the lexicographic optimisation in Step 3 is a $k$-objective efficient point. The revision to $f_{k}^{U B}$ is valid because Özlen and Azizoğlu [2009] show there cannot exist any other efficient point having objective function value $f_{k}(x)$ between $\max _{f \in S} f_{k}$ and the previous value of $f_{k}^{U B}$.

Note that $f_{i}^{A}$ always exists in Step 1 because $G$ is continuous, and because our invariants give $G\left(f_{1}^{L B}, \ldots, f_{i-1}^{L B}, f_{i}^{L B}, f_{i+1}^{L B}, \ldots, f_{k}^{L B}\right) \leq G^{B E S T}$; moreover, this $f_{i}^{A}$ is simple to find using standard univariate search techniques.

To prove that the algorithm terminates: Even if no bounds are updated in Steps 1 or 2, the procedure of Özlen and Azizoğlu [2009] will reduce the bound $f_{k}^{U B}$ in Step 3. This ensures that the bounds shrink during every loop through the algorithm, and because these shrinking bounds are integers we must terminate after finitely many steps.

To prove that the algorithm gives the optimal utility: Upon termination, either $G^{B E S T}$ is at least as good as anything obtainable within our bounds $f_{i}^{L B} \leq f_{i}(x) \leq f_{i}^{U B}$ (if we STOP in Step 1 or 2), or else these bounds have been reduced so far that the remaining integer efficient set is empty (if we STOP in Step 3). Either way, we know from our invariants that $G^{B E S T}$ is obtainable and that no better utility is possible.

Some final notes:

- In Step 2, hoping for an integer solution $x^{*}$ is optimistic. However, this simple test is cheap, and it can speed up the computation in the case where $x^{*} \in \mathbb{Z}$.

- Özlen and Azizoğlu [2009] implement Step 3 using $\epsilon$ multipliers for objectives, thereby transforming the lexicographic optimisation from Step 3 into a set of single-objective problems; see the paper for details. Other algorithms for generating nondominated objective vectors and/or efficient points may be used here instead of Özlen and Azizoğlu [2009] (for instance, in cases where highly efficient problem-specific algorithms are known).

- Although the MOIP problem in Step 3 is the computational bottleneck of the algorithm, it has only $k-1$ objectives, and we do not require all solutions (since we exit step 3 as soon as the bound $G^{B E S T}$ is updated). Both of these features make it significantly easier than the initial $k$-objective problem.

\section{An example problem}

In this section we illustrate our approach on a concrete example with $k=3$. This is a triobjective assignment problem of size $5 \times 5$, with the nonlinear utility function $G\left(f_{1}(x), f_{2}(x), f_{3}(x)\right)=$ $f_{1}(x)^{3}+f_{2}(x)^{3}+f_{3}(x)^{3}$. The individual objective coefficients for the problem, taken from Özlen and Azizoğlu [2009], are provided in Table 1.

The iterations of Algorithm 1 are summarised in Table 2. Columns in this table show the solutions of LP and IP problems in objective space, and the updated values of lower and upper bounds on the individual objective functions and the utility function as they appear in 
Tab. 1: Objective coefficients for the example problem

\begin{tabular}{|c|c|c|c|c|c|}
\hline$c_{1}$ & 1 & 2 & 3 & 4 & 5 \\
\hline 1 & 99 & 19 & 74 & 55 & 41 \\
\hline 2 & 23 & 81 & 93 & 39 & 49 \\
\hline 3 & 66 & 21 & 63 & 24 & 38 \\
\hline 4 & 65 & 41 & 7 & 39 & 66 \\
\hline 5 & 93 & 30 & 5 & 4 & 12 \\
\hline
\end{tabular}

\begin{tabular}{|c|c|c|c|c|c|}
\hline$c_{2}$ & 1 & 2 & 3 & 4 & 5 \\
\hline 1 & 28 & 39 & 19 & 42 & 7 \\
\hline 2 & 66 & 98 & 49 & 83 & 42 \\
\hline 3 & 73 & 26 & 42 & 13 & 54 \\
\hline 4 & 46 & 42 & 28 & 27 & 99 \\
\hline 5 & 80 & 17 & 99 & 59 & 68 \\
\hline
\end{tabular}

\begin{tabular}{|c|c|c|c|c|c|}
\hline$c_{3}$ & 1 & 2 & 3 & 4 & 5 \\
\hline 1 & 29 & 67 & 2 & 90 & 7 \\
\hline 2 & 84 & 37 & 64 & 64 & 87 \\
\hline 3 & 54 & 11 & 100 & 83 & 61 \\
\hline 4 & 75 & 63 & 69 & 96 & 3 \\
\hline 5 & 66 & 99 & 34 & 33 & 21 \\
\hline
\end{tabular}

Algorithm 1, For the columns representing bounds, an empty cell indicates that the value has not changed from the line above.

For the initialisation in Step 0 the procedure solves three lexicographic IPs, minimising objectives in the following lexicographic order: 1-2-3, 2-1-3, 3-1-2. There are of course many alternate methods of initialisation; we use lexicographic IPs here because they produce a good spread of nondominated objective vectors.

Step 1 then sets (and later updates) upper bounds on the individual objective functions based on the current best solution, $G^{B E S T}$. Step 2 updates the lower bounds by solving the linear programming relaxations with the upper bound constraints on the individual objective function values. Steps 1 and 2 are iterated for as long as they continue to update these lower and upper bounds. When the bounds cannot be updated further, Step 3 generates tri-objective nondominated objective vectors by generating a bi-objective nondominated set based on the upper bounds $f_{1}^{U B}, f_{2}^{U B}$ and $f_{3}^{U B}$. If Step 3 is able to improve upon the best utility value $G^{B E S T}$, it returns to Step 1; otherwise it updates $f_{3}^{U B}$ and returns to Step 2. In the final iteration, where Step 3 fails to find any feasible points within the current bounds, the entire algorithm terminates.

The optimal solution with $G(96,186,204)=15809256$ is identified at an early stage but it takes a large number of iterations to prove its optimality. We see from Table 2 that our shrinking bounds perform very well for this example: Algorithm 1 requires the solution of just eight IPs to find the optimal utility value. If we were to use the naïve method from the introduction and generate all nondominated objective vectors then we would require a total of 56 IPs to solve, as described in Özlen and Azizoğlu [2009]. This illustrates the way in which many IPs can be avoided (by eliminating nondominated objective vectors without explicitly generating them) using the shrinking bound techniques of Algorithm 1 .

\section{Conclusion}

In this study we propose a general algorithm to optimise a nonlinear utility function of multiple objectives over the integer efficient set. As an alternative to the naïve method of generating and evaluating all nondominated objective vectors, we restrict our search to a promising subset of nondominated vectors by computing and updating bounds on the individual objectives. The nondominated vectors within this promising subset are generated using the algorithm of Özlen and Azizoğlu [2009]. As illustrated by the example in Section 4, these bounding techniques can significantly reduce the total number of IPs to be solved. Because solving IPs is the most computationally expensive part of the algorithm, we expect these bounding techniques to yield a significant performance benefit for the algorithm as a whole.

For larger problems that remain too difficult to solve, Algorithm 1 can be used as an 


\begin{tabular}{|c|c|c|c|c|c|c|c|c|c|c|c|c|c|}
\hline Step & $\# \mathrm{IP}$ & & $f_{1}(x)$ & $f_{2}(x)$ & $f_{3}(x)$ & $G^{B E S T}$ & $f_{1}^{L B}$ & $f_{2}^{L B}$ & $f_{3}^{L B}$ & $G^{L B}$ & $f_{1}^{U B}$ & $f_{2}^{U B}$ & $f_{3}^{U B}$ \\
\hline 0 & 1 & IP $\operatorname{Min} f_{1}(x)$ & 86 & 214 & 324 & $44,448,624$ & 86 & $-\infty$ & $-\infty$ & 636,056 & $\infty$ & $\infty$ & $\infty$ \\
\hline 0 & 2 & IP Min $f_{2}(x)$ & 209 & 128 & 367 & & & 128 & & $2,733,208$ & & & \\
\hline 0 & 3 & IP $\operatorname{Min} f_{3}(x)$ & 291 & 348 & 129 & & & & 129 & $4,879,897$ & & & \\
\hline 1 & & Find $f_{1}^{A}, f_{2}^{A}, f_{3}^{A}$ & & & & & & & & & 342 & 346 & 346 \\
\hline 2 & & LP Min $f_{i}(x), i=1,2,3$ & 86 & 130.2 & 129.1 & & & 131 & 130 & $5,081,147$ & & & \\
\hline 1 & & Find $f_{1}^{A}, f_{2}^{A}, f_{3}^{A}$ & & & & & & & & & & & \\
\hline 3 & 4 & IP $\operatorname{Min} f_{1}(x), f_{2}(x)$ & 86 & 214 & 324 & & & & & & & & \\
\hline 3 & 5 & IP Min $f_{1}(x), f_{2}(x)$ & 96 & 186 & 204 & $15,809,256$ & & & & & & & \\
\hline 1 & & Find $f_{1}^{A}, f_{2}^{A}, f_{3}^{A}$ & & & & & & & & & 224 & 234 & 234 \\
\hline 2 & & LP $\operatorname{Min} f_{i}(x), i=1,2,3$ & 93.5 & 169.8 & 157.3 & & 94 & 170 & 158 & $9,687,896$ & & & \\
\hline 1 & & Find $f_{1}^{A}, f_{2}^{A}, f_{3}^{A}$ & & & & & & & & & 190 & 222 & 215 \\
\hline 2 & & LP $\operatorname{Min} f_{i}(x), i=1,2,3$ & 95.1 & 178.4 & 167.6 & & 96 & 179 & 168 & $11,361,707$ & & & \\
\hline 1 & & Find $f_{1}^{A}, f_{2}^{A}, f_{3}^{A}$ & & & & & & & & & 174 & 216 & 209 \\
\hline 2 & & $\mathrm{LP} \operatorname{Min} f_{i}(x), i=1,2,3$ & 95.6 & 181.3 & 173.7 & & & 182 & 174 & $12,181,328$ & & & \\
\hline 1 & & Find $f_{1}^{A}, f_{2}^{A}, f_{3}^{A}$ & & & & & & & & & 165 & 212 & 207 \\
\hline 2 & & LP $\operatorname{Min} f_{i}(x), i=1,2,3$ & 95.8 & 182.4 & 177.6 & & & 183 & 178 & $12,652,975$ & & & \\
\hline 1 & & Find $f_{1}^{A}, f_{2}^{A}, f_{3}^{A}$ & & & & & & & & & 159 & 210 & 206 \\
\hline 2 & & LP $\operatorname{Min} f_{i}(x), i=1,2,3$ & 95.8 & 183.1 & 179.7 & & & 184 & 180 & $12,946,240$ & & & \\
\hline 1 & & Find $f_{1}^{A}, f_{2}^{A}, f_{3}^{A}$ & & & & & & & & & 155 & 208 & \\
\hline 2 & & $\mathrm{LP} \operatorname{Min} f_{i}(x), i=1,2,3$ & 95.9 & 183.5 & 181.6 & & & & 182 & $13,142,808$ & & & \\
\hline 1 & & Find $f_{1}^{A}, f_{2}^{A}$ & & & & & & & & & 152 & 207 & \\
\hline 2 & & LP $\operatorname{Min} f_{i}(x), i=1,2,3$ & 95.9 & 183.7 & 182.6 & & & & 183 & $13,242,727$ & & & \\
\hline 1 & & Find $f_{1}^{A}, f_{2}^{A}$ & & & & & & & & & 151 & 206 & \\
\hline 2 & & LP $\operatorname{Min} f_{i}(x), i=1,2,3$ & 95.9 & 183.7 & 183.5 & & & & 184 & $13,343,744$ & & & \\
\hline 1 & & Find $f_{1}^{A}, f_{2}^{A}$ & & & & & & & & & 149 & 205 & \\
\hline 2 & & LP $\operatorname{Min} f_{i}(x), i=1,2,3$ & 95.9 & 183.7 & 184.4 & & & & 185 & $13,445,865$ & & & \\
\hline 1 & & Find $f_{1}^{A}, f_{2}^{A}$ & & & & & & & & & 148 & 204 & \\
\hline 2 & & $\mathrm{LP} \operatorname{Min} f_{i}(x), i=1,2,3$ & 95.9 & 183.8 & 185.3 & & & & 186 & $13,549,096$ & & & \\
\hline 1 & & Find $f_{1}^{A}, f_{2}^{A}$ & & & & & & & & & 146 & & \\
\hline 2 & & $\mathrm{LP} \operatorname{Min} f_{i}(x), i=1,2,3$ & & 183.9 & 185.5 & & & & & & & & \\
\hline 3 & 6 & IP Min $f_{1}(x), f_{2}(x)$ & 96 & 186 & 204 & & & & & & & & 203 \\
\hline 3 & 7 & IP $\operatorname{Min} f_{1}(x), f_{2}(x)$ & inf. & & & & & & & & & & \\
\hline 2 & & $\mathrm{LP} \operatorname{Min} f_{i}(x), i=1,2$ & 97.3 & 184.6 & & & 98 & 185 & & $13,707,673$ & & & \\
\hline 1 & & Find $f_{1}^{A}, f_{2}^{A}, f_{3}^{A}$ & & & & & & & & & 144 & 203 & \\
\hline 2 & & LP $\operatorname{Min} f_{i}(x), i=1,2,3$ & 97.3 & 184.7 & 186.4 & & & & 187 & $13,812,020$ & & & \\
\hline 1 & & Find $f_{1}^{A}, f_{2}^{A}$ & & & & & & & & & 143 & 202 & \\
\hline 2 & & LP $\operatorname{Min} f_{i}(x), i=1,2,3$ & 97.3 & 184.7 & 187.3 & & & & 188 & $13,917,489$ & & & \\
\hline 1 & & Find $f_{1}^{A}, f_{2}^{A}$ & & & & & & & & & 141 & 201 & \\
\hline 2 & & LP $\operatorname{Min} f_{i}(x), i=1,2,3$ & 97.3 & 184.8 & 188.3 & & & & 189 & $14,024,086$ & & & \\
\hline 1 & & Find $f_{1}^{A}, f_{2}^{A}$ & & & & & & & & & 139 & 200 & \\
\hline 2 & & LP $\operatorname{Min} f_{i}(x), i=1,2,3$ & 97.3 & 184.9 & 189.2 & & & & 190 & $14,141,817$ & & & \\
\hline 1 & & Find $f_{1}^{A}, f_{2}^{A}$ & & & & & & & & & 137 & & \\
\hline 2 & & LP Min $f_{i}(x), i=2,3$ & & 184.9 & 189.4 & & & & & & & & \\
\hline 3 & 8 & IP $\operatorname{Min} f_{1}(x), f_{2}(x)$ & inf. & & & $15,809,256$ & 98 & 185 & 190 & $14,131,817$ & 137 & 200 & 203 \\
\hline
\end{tabular}

Tab. 2: Iteration details of Algorithm 1 on the example problem instance 
approximation algorithm. We can terminate the algorithm at any time, whereupon $G^{B E S T}$ and $G\left(f_{1}^{L B}, f_{2}^{L B}, \ldots, f_{k}^{L B}\right)$ will give upper and lower bounds for the optimal utility, and we will have a feasible point $x \in X$ for which $G\left(f_{1}(x), f_{2}(x), \ldots, f_{k}(x)\right)=G^{B E S T}$.

We hope that this study stimulates future work in the field of multi-objective optimisation. One promising direction for future research may be to apply our algorithm to specific families of MOCO problems. The special structure of the constraints in these families might help to improve the efficiency of our algorithm for nonlinear utility functions.

\section{Acknowledgements}

The third author is supported by the Australian Research Council under the Discovery Projects funding scheme (project DP1094516). We thank the anonymous reviewers for their suggestions.

\section{References}

M. Abbas and D. Chaabane. Optimizing a linear function over an integer efficient set. European J. Oper. Res., 174(2):1140-1161, 2006.

H. P. Benson and E. Sun. Outcome space partition of the weight set in multiobjective linear programming. J. Optim. Theory Appl., 105(1):17-36, 2000.

H. P. Benson and E. Sun. A weight set decomposition algorithm for finding all efficient extreme points in the outcome set of a multiple objective linear program. European J. Oper. Res., 139(1):26-41, 2002.

C. Dhaenens, J. Lemesre, and E.G. Talbi. K-PPM: a new exact method to solve multi-objective combinatorial optimization problems. European J. Oper. Res., 200(1):45-53, 2010.

M. Ehrgott. A discussion of scalarization techniques for multiple objective integer programming. Ann. Oper. Res., 147:343-360, 2006.

M. Ehrgott and X. Gandibleux. A survey and annotated bibliography of multiobjective combinatorial optimization. OR Spektrum, 22(4):425-460, 2000.

M. Ehrgott and X. Gandibleux, editors. Multiple criteria optimization: state of the art annotated bibliographic surveys. International Series in Operations Research \& Management Science, 52. Kluwer Academic Publishers, Boston, MA, 2002.

M. Ehrgott and X. Gandibleux. Approximative solution methods for multiobjective combinatorial optimization. Top, 12(1):1-89, 2004.

L. Galand, P. Perny, and O. Spanjaard. Choquet-based optimisation in multiobjective shortest path and spanning tree problems. European Journal of Operational Research, 204(2):303 315, 2010a.

L. Galand, P. Perny, and O. Spanjaard. A branch and bound algorithm for choquet optimization in multicriteria problems. In M. Ehrgott, B. Naujoks, T. J. Stewart, and J. Wallenius, 
editors, Multiple Criteria Decision Making for Sustainable Energy and Transportation Systems, volume 634 of Lecture Notes in Economics and Mathematical Systems, pages 355-365. Springer Berlin Heidelberg, 2010b.

J.M. Jorge. An algorithm for optimizing a linear function over an integer efficient set. European J. Oper. Res., 195(1):98-103, 2009.

K. Klamroth, J. Tind, and S. Zust. Integer programming duality in multiple objective programming. J. Global Optim., 29(1):1-18, 2004.

D. Klein and E. Hannan. An algorithm for the multiple objective integer linear programming problem. European J. Oper. Res., 9(4):378-385, 1982.

F. Le Huédé, M. Grabisch, C. Labreuche, and P. Savéant. Integration and propagation of a multi-criteria decision making model in constraint programming. Journal of Heuristics, 12: 329-346, 2006.

M. Özlen and M. Azizoğlu. Multi-objective integer programming: a general approach for generating all non-dominated solutions. European J. Oper. Res., 199(1):25-35, 2009.

Ö. Özpeynirci and M. Köksalan. An exact algorithm for finding extreme supported nondominated points of multiobjective mixed integer programs. Management Science, 56(12): 2302-2315, 2010.

P. Perny and O. Spanjaard. A preference-based approach to spanning trees and shortest paths problems. European Journal of Operational Research, 162(3):584 - 601, 2005.

A. Przybylski, X. Gandibleux, and M. Ehrgott. A two phase method for multi-objective integer programming and its application to the assignment problem with three objectives. Discrete Optim., 7(3):149-165, 2010a.

A. Przybylski, X. Gandibleux, and M. Ehrgott. A recursive algorithm for finding all nondominated extreme points in the outcome set of a multiobjective integer programme. INFORMS J. Comput., 22(3):371-386, 2010b.

J. Sylva and A. Crema. A method for finding the set of non-dominated vectors for multiple objective integer linear programs. European J. Oper. Res., 158(1):46-55, 2004.

Melih Ozlen

School of Mathematical and Geospatial Sciences, RMIT University

GPO Box 2476V, Melbourne VIC 3001, Australia

(melih.ozlen@rmit.edu.au)

Meral Azizoğlu

Department of Industrial Engineering, Middle East Technical University

Ankara 06531, Turkey

(meral@ie.metu.edu.tr) 
Benjamin A. Burton

School of Mathematics and Physics, The University of Queensland Brisbane QLD 4072, Australia

(bab@maths.uq.edu.au) 